\title{
The Mixed Degree of Families of Lattice Polytopes
}

\author{
Benjamin Nill
}

\begin{abstract}
The degree of a lattice polytope is a notion in Ehrhart theory that was studied quite intensively over previous years. It is well known that a lattice polytope has normalized volume one if and only if its degree is zero. Recently, Esterov and Gusev gave a complete classification result for families of $n$ lattice polytopes in $\mathbb{R}^{n}$ whose mixed volume equals one. Here, we give a reformulation of their result involving the novel notion of mixed degree that generalizes the degree similar to how the mixed volume generalizes the volume. We discuss and motivate this terminology, also from an algebro-geometric viewpoint, and explain why it extends a previous definition of Soprunov. We also remark how a recent combinatorial result due to Bihan solves a related problem posed by Soprunov.
\end{abstract}

Keywords. Mixed degree, Mixed volume, Ehrhart polynomials, Lattice polytopes.

\section{Definitions and Motivation}

\subsection{Introduction}

Lattice polytopes in $\mathbb{R}^{n}$ are called hollow (or lattice-free) $[8,24]$ if they have no lattice points (i.e., elements in $\mathbb{Z}^{n}$ ) in their relative interiors. In this paper, we initiate the study of large families of lattice polytopes with hollow Minkowski sums. We observe that such a family can consist of at most $n$ elements (Proposition 4). In Theorem 5, we deduce from the main result in [15] that a family of $n$ lattice polytopes in $\mathbb{R}^{n}$ has mixed volume one if and only if the Minkowski sums of all subfamilies are hollow. To measure the 'hollowness' of a family of lattice polytopes, we introduce the mixed degree of a family of lattice polytopes. It naturally generalizes the much-studied notion of the degree of a lattice polytope in a manner similar to how the mixed volume generalizes the normalized volume (see Sect. 1.3). Our goal is to convince the reader that the mixed degree is worthwhile to study invariant of a family of lattice polytopes. As a first positive evidence, we note its nonnegativity (Sect. 2.1), a generalization 
of the nonnegativity of the degree, and prove the characterization of mixed degree zero by mixed volume one (Sect. 2.2) in analogy to the characterization of degree zero by normalized volume one. We will also explain how the definition given here generalizes an independent definition of Soprunov, and we give a new combinatorial proof of his lower bound theorem (see Sect. 2.3).

\subsection{Basic Definitions}

Let us recall that a lattice polytope $P \subset \mathbb{R}^{n}$ is a polytope whose vertices are elements of the lattice $\mathbb{Z}^{n}$. Two lattice polytopes are unimodularly equivalent if they are isomorphic via an affine lattice-preserving transformation. We denote by $\operatorname{conv}(A)$ the convex hull of a set $A \subseteq \mathbb{R}^{n}$. We say that $P$ is an $n$-dimensional unimodular simplex if it is unimodularly equivalent to $\Delta_{n}:=\operatorname{conv}\left(0, e_{1}, \ldots, e_{n}\right)$, where 0 denotes the origin of $\mathbb{R}^{n}$ and $e_{1}, \ldots, e_{n}$ the standard basis vectors. We define the normalized volume $\operatorname{Vol}(P)$ as $\operatorname{dim}(P)$ ! times the Euclidean volume with respect to the affine lattice given by the intersection of $\mathbb{Z}^{n}$ and the affine span of $P$. Note that $\operatorname{Vol}\left(\Delta_{n}\right)=1$.

Definition 1. Let $P_{1}, \ldots, P_{m} \subset \mathbb{R}^{n}$ be a finite set of lattice polytopes.

- For $k \in \mathbb{Z}_{\geq 1}$ we set $[k]:=\{1, \ldots, k\}$.

- For $\emptyset \neq I \subseteq[m]$ we define their Minkowski sum

$$
P_{I}:=\sum_{i \in I} P_{i}:=\left\{\sum_{i \in I} x_{i}: x_{i} \in P_{i} \text { for } i \in I\right\} .
$$

We set $P_{\emptyset}:=\{0\}$.

- For $\emptyset \neq A \subset \mathbb{R}^{n}$ we define

$$
A_{\mathbb{Z}}:=A \cap \mathbb{Z}^{n}, \quad \operatorname{int}_{\mathbb{Z}}(A):=\operatorname{int}(A) \cap \mathbb{Z}^{n},
$$

where the interior always denotes the relative interior (i.e., the interior with respect to the affine span of $A$ ). Recall that the interior of a point is considered to be the point itself, i.e., it is non-empty.

- For convenience, we say $P_{1}, \ldots, P_{m}$ is proper in $\mathbb{R}^{n}$ if

$$
\operatorname{dim}\left(P_{1}\right) \geq 1, \ldots, \operatorname{dim}\left(P_{m}\right) \geq 1, \quad \operatorname{dim}\left(P_{[m]}\right)=n .
$$

One of the reasons for excluding points in a proper family is that adding a point to a family just results in a lattice translation of their Minkowski sum.

Throughout the paper, we identify two families of lattice polytopes if they agree up to a simultaneous unimodular transformation of $\mathbb{Z}^{n}$, permutations of the factors, and (lattice) translations of the factors.

Let us state our main definition.

Definition 2. Let $P_{1}, \ldots, P_{m} \subset \mathbb{R}^{n}$ be a finite set of lattice polytopes.

- We define the mixed codegree of $P_{1}, \ldots, P_{m}$ as follows:

- If there exists $\emptyset \neq I \subseteq[m]$ such that $\operatorname{int}_{\mathbb{Z}}\left(P_{I}\right) \neq \emptyset$, then $\operatorname{mcd}\left(P_{1}, \ldots, P_{m}\right)$ is defined as the minimal cardinality of such $I$;

- otherwise, $\operatorname{mcd}\left(P_{1}, \ldots, P_{m}\right):=m+1$. 
Let us note that

$$
1 \leq \operatorname{mcd}\left(P_{1}, \ldots, P_{m}\right) \leq m+1 .
$$

- We define the mixed degree of $P_{1}, \ldots, P_{m}$ as

$$
\operatorname{md}\left(P_{1}, \ldots, P_{m}\right):=\operatorname{dim}\left(P_{[m]}\right)+1-\operatorname{mcd}\left(P_{1}, \ldots, P_{m}\right) .
$$

Note that a family where one of the lattice polytopes is a point automatically has mixed degree equal to the dimension of $P_{[m]}$. We remark that for a proper family

$$
n-m \leq \operatorname{md}\left(P_{1}, \ldots, P_{m}\right) \leq n .
$$

Example 3. Consider the following family of hollow lattice polytopes in $\mathbb{R}^{2}$ :

$$
P_{1}=\operatorname{conv}\left(0, e_{1}\right), \quad P_{2}=\operatorname{conv}\left(0, e_{2}\right), \quad P_{3}=\operatorname{conv}\left(0, e_{1}, e_{2}, e_{1}+e_{2}\right),
$$

where $e_{1}, e_{2}$ is the standard basis of $\mathbb{R}^{2}$. Then, the Minkowski sums of any two of these three lattice polytopes are hollow, while the Minkowski sum of all three is not. Hence, $\operatorname{mcd}\left(P_{1}, P_{2}, P_{3}\right)=3$ and $\operatorname{md}\left(P_{1}, P_{2}, P_{3}\right)=0$.

\subsection{Relation to the Ehrhart-Theoretic Degree}

Let us explain where the definition of the mixed (co-)degree comes from. Let $P \subset \mathbb{R}^{n}$ be an $n$-dimensional lattice polytope. The codegree of $P$ is defined as the smallest positive $k$ such that $\operatorname{int}_{\mathbb{Z}}(k P) \neq \emptyset$, and the degree of $P$ is given as $n+1-\operatorname{codeg}(P)$. Hence, for $P_{1}:=P, \ldots, P_{n}:=P$, we see ${ }^{1}$ that

$$
\operatorname{mcd}\left(P_{1}, \ldots, P_{n}\right)=\operatorname{codeg}(P), \quad \operatorname{md}\left(P_{1}, \ldots, P_{n}\right)=\operatorname{deg}(P) .
$$

This unmixed situation has been studied rather intensively (e.g., [4,17,22]) leading to applications and relations to the adjunction theory of polarized toric varieties $[1,12,13]$, dual defective toric varieties [14], and almost-neighborly point configurations [23]. We hope to eventually generalize some of the achieved results to the mixed situation.

Note that the degree of a lattice polytope $P$ is originally defined as the degree of the $h^{*}$-polynomial $h_{P}^{*}$, the numerator polynomial of the rational generating function of the Ehrhart polynomial of $P$ (e.g., [4]). In this case, the relation between degree and codegree follows from Ehrhart-Macdonald reciprocity (see [4, Remark 1.2]). We remark that a priori there are several possibilities for how to define a generalization of the degree to families of lattice polytopes. Here, we generalize the geometric notion of codegree instead of the more algebraic definition of degree. It would be very interesting to find an analogous natural interpretation for the mixed degree. Originally motivated by tropical geometry [27], there is current research to investigate a mixed version of the $h^{*}$-polynomial $[16,18]$. However, its properties are yet to be fully understood. We caution the reader that the degree of the mixed $h^{*}$-polynomial as defined in [16] is in general not equal to the mixed degree discussed here. For instance, for the one-element family $P_{1}:=P$ with $m=1$, the mixed degree equals $n$ or $n-1$ depending on whether $P$ has interior lattice points or not;

\footnotetext{
${ }^{1}$ We warn the reader that $\operatorname{mcd}(P)$ is in general different from $\operatorname{codeg}(P)$, as well as $\operatorname{mcd}\left(P_{1}, \ldots, P_{m}\right)$ is in general different from $\operatorname{codeg}\left(P_{1} * \cdots * P_{m}\right)$ (see Definition 15).
} 
while on the other hand, equation $[16,(10)]$ implies that the degree of the mixed $h^{*}$-polynomial is in this case always equal to $n$ if $n$ is odd.

\subsection{Motivation from Algebraic Geometry}

Given a proper family of lattice polytopes, it is natural to consider the following situation. We say $P_{1}, \ldots, P_{m}$ is irreducible if $\operatorname{int}_{\mathbb{Z}}\left(P_{I}\right)=\emptyset$ for any $\emptyset \neq I \subsetneq[m]$ and $\operatorname{int}_{\mathbb{Z}}\left(P_{[m]}\right) \neq \emptyset$. The study of irreducible families of given mixed degree turns up in the Batyrev-Borisov construction of mirror-symmetric CalabiYau complete intersections $[2,3,5]$. For this, let us call a proper family of lattice polytopes $P_{1}, \ldots, P_{m}$ a reflexive family if their Minkowski sum is a reflexive polytope (up to translation), e.g., a so-called nef-partition [3]. In this case, let us choose generic Laurent polynomials $f_{1}, \ldots, f_{m}$ with Newton polytopes $P_{1}, \ldots, P_{m}$. Then, the complete intersection $V$ of the closures of the hypersurfaces $\left\{f_{i}=0\right\} \subset\left(\mathbb{C}^{*}\right)^{n}$ in the toric Gorenstein Fano variety associated with $P_{[m]}$ is a Calabi-Yau variety of dimension $n-m$ if $V$ is not empty (see $[2,3])$. Let us assume that the reflexive family is irreducible. In this case, $[3$, Corollary 3.5] implies that the dimension of $V$ equals the mixed degree minus one; $V$ is non-empty if and only if the mixed degree is at least one; and $V$ is an irreducible variety if and only if the mixed degree is at least two. By the so-called semi-simplicity principle for nef-partitions ([3, Section 5] and more generally [5, Proposition 6.13]), any reflexive family can be partitioned into irreducible reflexive subfamilies. Hence, in this toric setting, the study of Calabi-Yau complete intersections of given dimension is closely related to the study of irreducible families of given mixed degree.

\subsection{Structure of the Paper}

Section 2 contains the main results of this paper. Proofs are give in Sect. 3 .

\section{Results on the Mixed Degree}

In this section, we describe our results on the mixed degree of a family of lattice polytopes. We will postpone all proofs to Sect. 3.

\subsection{Nonnegativity}

Here is our first observation. It can be deduced from [10, Corollary 2.3] by Cox and Dickenstein which relies on quite deep spectral sequence arguments. We will provide an alternative self-contained, short and elementary proof.

Proposition 4. The mixed degree is nonnegative.

Let us recall how one can convex-geometrically prove nonnegativity in the unmixed situation. Note that for an arbitrary interior point of an $n$-dimensional lattice polytope $P$, Carathéodory's theorem allows to find vertices $v_{0}, \ldots, v_{n}$ of $P$ such that the point is in the convex hull of these vertices. Therefore, also $\left(v_{0}+\cdots+v_{n}\right) /(n+1)$ is in the interior of $P$. Hence, $\operatorname{int}_{\mathbb{Z}}((n+1) P) \neq \emptyset$, thus, $\operatorname{codeg}(P) \leq n+1$, so $\operatorname{deg}(P) \geq 0$. Hence, Proposition 4 may be seen as a mixed version of Carathéodory's theorem in the following sense: Given $P_{1}, \ldots, P_{m}$ lattice polytopes in $\mathbb{R}^{n}$ with $m>n$, there exists a non-empty subset 
$I \subseteq[m]$ of cardinality $|I| \leq n+1$ such that the Minkowski sum $P_{I}$ contains a lattice point in its relative interior.

\subsection{Mixed Degree Zero}

In the unmixed case, $\operatorname{deg}(P)=0$ if and only if $\operatorname{Vol}(P)=1$ (see [4]). As we will see, the analogous statement is also true in the mixed situation. This may be regarded as favorable evidence that the definition of the mixed degree is a reasonable generalization of the unmixed degree of a lattice polytope.

For this, let us define the (normalized) mixed volume $\mathrm{MV}\left(P_{1}, \ldots, P_{n}\right)$ of a family $P_{1}, \ldots, P_{n} \subset \mathbb{R}^{n}$ as the coefficient of $\lambda_{1} \cdots \lambda_{n}$ of the homogeneous polynomial $\operatorname{vol}_{n}\left(\lambda_{1} P_{1}+\cdots+\lambda_{n} P_{n}\right)$, where $\operatorname{vol}_{n}$ is the standard Euclidean volume of $\mathbb{R}^{n}$, see $[15,25]$. It is nonnegative, monotone with respect to inclusion, and multilinear. Note that the mixed volume defined here is normalized such that for an $n$-dimensional lattice polytope $P \subset \mathbb{R}^{n}$, we have $\operatorname{MV}(P, \ldots, P)=\operatorname{Vol}(P)$. Hence, the following result generalizes the unmixed statement.

Theorem 5. Let $P_{1}, \ldots, P_{n}$ be a proper family of lattice polytopes in $\mathbb{R}^{n}$. Then, the following conditions are equivalent:

1. $\operatorname{md}\left(P_{1}, \ldots, P_{n}\right)=0$

2. $\operatorname{MV}\left(P_{1}, \ldots, P_{n}\right)=1$.

While the implication $(1) \Rightarrow(2)$ has a short proof, the reverse implication $(2) \Rightarrow(1)$ relies on the highly non-trivial classification of $n$ lattice polytopes of mixed volume one by Esterov and Gusev [15]. It would be desirable to find a direct, classification-free proof.

In the unmixed case, there is only one $n$-dimensional lattice polytope of degree 0 , respectively normalized volume 1 , namely, the unimodular $n$-simplex. Such a uniqueness result also holds in the mixed case if all lattice polytopes in the family are full-dimensional. This is essentially a corollary to [9, Prop. 2.7].

Proposition 6. Let $P_{1}, \ldots, P_{m}$ be $n$-dimensional lattice polytopes in $\mathbb{R}^{n}$. Then $\operatorname{md}\left(P_{1}, \ldots, P_{m}\right)=0$ if and only if $m \geq n$ and $P_{1}, \ldots, P_{m}$ equal the same unimodular $n$-simplex (up to translations).

In the low-dimensional case, the situation is more complicated. From the results of Esterov and Gustev [15], we get an inductive description of families of $n$ lattice polytopes of mixed degree zero. For this, let us define for $\emptyset \neq I \subseteq[m]$ the lattice projection $\pi_{I}$ along the affine span of $P_{I}$. More precisely, $\pi_{I}$ is the $\mathbb{R}$-linear map induced by the lattice surjection $\mathbb{Z}^{n} \rightarrow \mathbb{Z}^{n} / \Gamma$, where $\Gamma$ is the subgroup that is a translate of the set of lattice points in the affine hull of $P_{I}$.

Corollary 7. Let $P_{1}, \ldots, P_{n}$ be a proper family of lattice polytopes in $\mathbb{R}^{n}$. Then, $\operatorname{md}\left(P_{1}, \ldots, P_{n}\right)=0$ if and only if one of the following two cases holds:

1. $P_{1}, \ldots, P_{n}$ are contained in the same unimodular $n$-simplex (up to translations),

2. there exists an integer $1 \leq k<n$ such that (up to translations and permutation of $\left.P_{1}, \ldots, P_{n}\right) P_{1}, \ldots, P_{k}$ are contained in a $k$-dimensional subspace of $\mathbb{R}^{n}$ with $\operatorname{dim}\left(P_{[k]}\right)=k$ such that $\operatorname{md}\left(P_{1}, \ldots, P_{k}\right)=0$ and $\operatorname{md}\left(\pi_{[k]}\left(P_{k+1}\right), \ldots, \pi_{[k]}\left(P_{n}\right)\right)=0$. 
For $m>n$ we do not yet have such a complete classification result for all families of $m$ lattice polytopes in $\mathbb{R}^{n}$ of mixed degree 0 . However, we can show that there are essentially only finitely many cases.

Theorem 8. Let $P_{1}, \ldots, P_{m}$ be a proper family of lattice polytopes in $\mathbb{R}^{n}$ with $\operatorname{md}\left(P_{1}, \ldots, P_{m}\right)=0$ and $m>n$. Then, one of the following two cases holds:

1. $P_{1}, \ldots, P_{m}$ are contained in a unimodular $n$-simplex $Q$ (up to translations),

2. the family $P_{1}, \ldots, P_{m}$ belongs to a finite number of exceptions (whose number depends only on $n$ ).

Moreover, in the first case, at most $\left(2^{n}-1\right)(n-1)$ of the polytopes in the family are not equal to $Q$ (up to translations). More precisely, no face of $Q$ of dimension $j<n$ appears among $P_{1}, \ldots, P_{m}$ more than $j$ times (up to translations).

Remark 9. We leave it as an exercise to the reader to show that for $n=2$, there is precisely one exception in Theorem 8, namely, the family given in Example 3. It would be interesting to know whether there are exceptional families in Theorem 8 of length larger than $n+1$.

\subsection{Mixed Degree at Most One}

The following lower bound theorem can be found in [6] based upon [26].

Theorem 10. (Soprunov '07) Let $P_{1}, \ldots, P_{n}$ be $n$-dimensional lattice polytopes. Then

$$
\left|\operatorname{int}_{\mathbb{Z}}\left(P_{[n]}\right)\right| \geq \operatorname{MV}\left(P_{1}, \ldots, P_{n}\right)-1 .
$$

The original proof of Theorem 10 involved the Euler-Jacobi Theorem and Bernstein's Theorem. In [6, Problem 1], Soprunov asked whether there is a purely combinatorial proof. We can affirmatively answer this question in Sect. 3.4 by reducing it to a recent result by Bihan related to the nonnegativity of the so-called discrete mixed volume [7].

Example 11. Note that the full-dimensionality assumption in Soprunov's lower bound theorem cannot be removed. Consider in $\mathbb{R}^{2}$ a unimodular 2-simplex $P_{1}$ and a line segment $P_{2}$ parallel to one of the edges of $P_{1}$. If $P_{2}$ contains $k$ lattice points, then $\operatorname{MV}\left(P_{1}, P_{2}\right)=k-1$, while $\left|\operatorname{int}_{\mathbb{Z}}\left(P_{1}+P_{2}\right)\right|=0$.

In the unmixed case $\left(P_{1}=\cdots=P_{n}=P\right)$, Theorem 10 follows directly from Ehrhart theory, see [6]. Moreover, Soprunov observes in his note that equality is attained if and only if $\operatorname{deg}(P) \leq 1$. This observation led him to define in [6] a family of $n$-dimensional lattice polytopes $P_{1}, \ldots, P_{n}$ as having mixed degree at most 1 if equality in Theorem 10 is attained, and mixed degree 0 if $P_{[n]}$ has no interior lattice points. As the following result shows, this is compatible with our definition.

Proposition 12. Let $P_{1}, \ldots, P_{n}$ be $n$-dimensional lattice polytopes. Then $\left|\operatorname{int}_{\mathbb{Z}}\left(P_{[n]}\right)\right|=\operatorname{MV}\left(P_{1}, \ldots, P_{n}\right)-1$ if and only if $\operatorname{md}\left(P_{1}, \ldots, P_{n}\right) \leq 1$. 


\section{Proofs}

\subsection{Nonnegativity}

This will be a simple consequence of basic properties of the mixed volume. For this, let us recall a well-known alternative formula (going at least implicitly back to [20], it can be found in this explicit form e.g. in [19, Prop. 5.2]).

Proposition 13. (Khovanskii '78) Let $P_{1}, \ldots, P_{n}$ be lattice polytopes in $\mathbb{R}^{n}$. Then

$$
\operatorname{MV}\left(P_{1}, \ldots, P_{n}\right)=\sum_{I \subseteq[n]}(-1)^{n-|I|}\left|P_{I} \cap \mathbb{Z}^{n}\right|
$$

Recall that $\left|P_{\emptyset} \cap \mathbb{Z}^{n}\right|=1$. Using reciprocity, one gets another slightly less well-known formula involving interior lattice points (cf. [20]).

Corollary 14. Let $P_{1}, \ldots, P_{n}$ be lattice polytopes in $\mathbb{R}^{n}$. Then

$$
\operatorname{MV}\left(P_{1}, \ldots, P_{n}\right)=1+\sum_{\emptyset \neq I \subseteq[n]}(-1)^{\operatorname{dim}\left(P_{I}\right)-|I|}\left|\operatorname{int}_{\mathbb{Z}}\left(P_{I}\right)\right|
$$

In particular, if $P_{1}, \ldots, P_{n}$ are $n$-dimensional, then

$$
\operatorname{MV}\left(P_{1}, \ldots, P_{n}\right)=1+\sum_{\emptyset \neq I \subseteq[n]}(-1)^{n-|I|}\left|\operatorname{int}_{\mathbb{Z}}\left(P_{I}\right)\right| .
$$

Proof. Let us denote by $\operatorname{ehr}_{P}(t)$ the Ehrhart polynomial of a lattice polytope $P \subset \mathbb{R}^{n}$, i.e., $\operatorname{ehr}_{P}(t)=\left|(t P) \cap \mathbb{Z}^{n}\right|$ for $t \in \mathbb{Z}_{\geq 1}$. Ehrhart-Macdonald reciprocity yields

$$
\operatorname{ehr}_{P}(-1)=(-1)^{\operatorname{dim}(P)}\left|\operatorname{int}_{\mathbb{Z}}(P)\right|
$$

(For the case of dimension 0, recall that the interior of a lattice point is the lattice point itself.) Applying Proposition 13 to $t P_{1}, \ldots, t P_{n}$ for $t \in \mathbb{Z}_{\geq 1}$ gives

$$
t^{n} \operatorname{MV}\left(P_{1}, \ldots, P_{n}\right)=\operatorname{MV}\left(t P_{1}, \ldots, t P_{n}\right)=(-1)^{n}+\sum_{\emptyset \neq I \subseteq[n]}(-1)^{n-|I|} \operatorname{ehr}_{P_{I}}(t) .
$$

Plugging in $t=-1$, then Ehrhart-Macdonald reciprocity yields

$$
(-1)^{n} \operatorname{MV}\left(P_{1}, \ldots, P_{n}\right)=(-1)^{n}+\sum_{\emptyset \neq I \subseteq[n]}(-1)^{n-|I|}(-1)^{\operatorname{dim}\left(P_{I}\right)}\left|\operatorname{int}_{\mathbb{Z}}\left(P_{I}\right)\right|
$$

Proof of Proposition 4. It follows from the definition of the mixed degree that it suffices to show nonnegativity for a proper family $P_{1}, \ldots, P_{n+1}$ in $\mathbb{R}^{n}$. We assume that $\operatorname{int}_{\mathbb{Z}}\left(P_{I}\right)=\emptyset$ for any $\emptyset \neq I \subseteq[n+1]$. Consider the proper family $P_{1}, \ldots, P_{n-1}, P_{n}+P_{n+1}$. Corollary 14 and multilinearity of the mixed volume yield that $1=\operatorname{MV}\left(P_{1}, \ldots, P_{n-1}, P_{n}+P_{n+1}\right)=\operatorname{MV}\left(P_{1}, \ldots, P_{n-1}, P_{n}\right)+$ $\operatorname{MV}\left(P_{1}, \ldots, P_{n-1}, P_{n+1}\right)$. However, Corollary 14 also implies that both of these summands equal 1 , a contradiction. 


\subsection{Mixed Degree 0: The Full-Dimensional Case}

Since it might be of independent interest, we provide several characterizations of this situation. For this, let us recall the following definition.

Definition 15. The Cayley polytope of lattice polytopes $P_{1}, \ldots, P_{m}$ in $\mathbb{R}^{n}$ is defined as

$$
P_{1} * \cdots * P_{m}:=\operatorname{conv}\left(P_{1} \times\left\{e_{1}\right\}, \ldots, P_{m} \times\left\{e_{m}\right\}\right) \subset \mathbb{R}^{n+m}
$$

where $e_{1}, \ldots, e_{m}$ is the standard basis of of $\mathbb{R}^{m}$. Note that if $\operatorname{dim}\left(P_{[m]}\right)=n$, then

$$
\operatorname{dim}\left(P_{1} * \cdots * P_{m}\right)=n+m-1 .
$$

Proposition 16. Let $P_{1}, \ldots, P_{n}$ be $n$-dimensional lattice polytopes in $\mathbb{R}^{n}$. Then, the following conditions are equivalent:

1. $\operatorname{md}\left(P_{1}, \ldots, P_{n}\right)=0$

2. $\operatorname{int}_{\mathbb{Z}}\left(P_{1}+\cdots+P_{n}\right)=\emptyset$

3. $\operatorname{MV}\left(P_{1}, \ldots, P_{n}\right)=1$

4. $P_{1}, \ldots, P_{n}$ are lattice translates of the same unimodular $n$-simplex

5. $\operatorname{Vol}\left(P_{1}+\cdots+P_{n}\right)=n^{n}$, which is the minimal possible value

6. $\operatorname{Vol}\left(P_{1} * \cdots * P_{n}\right)=\left(\begin{array}{c}2 n-1 \\ n\end{array}\right)$, which is the minimal possible value

7. $\operatorname{deg}\left(P_{1} * \cdots * P_{n}\right)=n-1$, which is the minimal possible value

We remark that otherwise $\operatorname{deg}\left(P_{1} * \cdots * P_{n}\right)=n$.

Proof. (1) $\Leftrightarrow$ (2) follows from full-dimensionality. (1) $\Rightarrow$ (3) by Corollary 14. $(3) \Rightarrow(4)$ was proven in [9, Prop. 2.7]. Clearly, (4) $\Rightarrow(1)$.

$(5) \Rightarrow(3)$ follows from an expression in terms of multinomial coefficients (e.g., $[25])$ :

$$
\operatorname{Vol}\left(P_{1}+\cdots+P_{n}\right)=\sum_{k_{1}+\cdots+k_{n}=n}\left(\begin{array}{c}
n \\
k_{1}, \cdots, k_{n}
\end{array}\right) \cdot \operatorname{MV}\left(P_{1}^{\left(k_{1}\right)}, \ldots, P_{n}^{\left(k_{n}\right)}\right),
$$

where the sum is over all nonnegative integer $n$-tuples $k_{1}, \ldots, k_{n}$ satisfying the condition $k_{1}+\cdots+k_{n}=n$; moreover, $P_{i}^{\left(k_{i}\right)}$ means that $P_{i}$ should be repeated $k_{i}$ times. Since all lattice polytopes are full-dimensional, each of the mixed volumes in the sum is positive. Let us note that, if they are all equal to 1 , the right side equals $n^{n}$. For $(3) \Rightarrow(5)$ note that if $\operatorname{MV}\left(P_{1}, \ldots, P_{n}\right)=1$, then also each of the mixed volumes (since they are all positive) must be equal to 1 by the Alexandrov-Fenchel inequality (e.g., [25]). Alternatively, one can directly verify $(4) \Rightarrow(5)$.

$(6) \Rightarrow(4)$ uses the following formula (e.g., [11]): $\operatorname{Vol}\left(P_{1} * \cdots * P_{n}\right)$ equals the sum of $\operatorname{MV}\left(P_{i_{1}}, \ldots, P_{i_{n}}\right)$ over all possible choices of unordered $n$-tuples $i_{1}, \ldots, i_{n} \in[n]$, where repetitions are allowed (there are $\left(\begin{array}{c}2 n-1 \\ n\end{array}\right)$ such choices). Since all lattice polytopes are full-dimensional, each of the mixed volumes in the sum is positive. The converse $(3) \Rightarrow(6)$ follows as above from the Alexandrov-Fenchel inequality or directly by checking $(4) \Rightarrow(6)$.

$(2) \Leftrightarrow(7)$ is a consequence of the so-called Cayley-Trick. Consider the lattice projection $\pi$ mapping $P_{1} * \cdots * P_{n}$ onto $\Delta_{n-1}$. Therefore, $\operatorname{codeg}\left(P_{1} *\right.$ $\left.\ldots * P_{n}\right) \geq \operatorname{codeg}\left(\Delta_{n-1}\right)=n$. Now, the intersection of $n\left(P_{1} * \cdots * P_{n}\right)$ with 
the preimage of the unique interior lattice point in $n \Delta_{n-1}$ is unimodularly equivalent to $P_{1}+\cdots+P_{n}$. Therefore, we have $\operatorname{codeg}\left(P_{1} * \cdots * P_{n}\right)>n$ (or equivalently, $\left.\operatorname{deg}\left(P_{1} * \cdots * P_{n}\right)<n\right)$ precisely when $(2)$ is satisfied. Let us note that in this case, by (4), $P_{1} * \cdots * P_{n} \cong \Delta_{n} \times \Delta_{n-1}$, so its degree equals $n-1$.

Proof of Proposition 6. The implication follows from (1) and applying 1. $\Rightarrow 4$. in Proposition 16 to any subfamily of $n$ lattice polytopes. The reverse implication is a direct consequence of $\operatorname{codeg}\left(\Delta_{n}\right)=n+1$.

\subsection{Mixed Degree 0: The Low-Dimensional Case}

We used before that the mixed volume of full-dimensional polytopes is positive. Bernstein's criterion (described in [20], we also refer to [25, Theorem 5.1.8]) gives the precise generalization.

Lemma 17 (Bernstein-Khovanskii '78). $\mathrm{MV}\left(P_{1}, \ldots, P_{n}\right) \geq 1$ if and only if $\operatorname{dim}\left(P_{I}\right) \geq|I|$ for all $\emptyset \neq I \subseteq[n]$.

Note that in this case $P_{1}, \ldots, P_{n}$ is necessarily a proper family. Let us also recall the following well-known fact about mixed volumes (this is [15, Prop. 2], we refer also to [25, Theorem 5.3.1]).

Lemma 18 (Esterov, Gusev '12). Let $P_{1}, \ldots, P_{n}$ be lattice polytopes in $\mathbb{R}^{n}$. If $P_{1}, \ldots, P_{k}$ (for $\left.1 \leq k \leq n\right)$ are contained in a $k$-dimensional rational subspace $L$ of $\mathbb{R}^{n}$, then

$$
\operatorname{MV}\left(P_{1}, \ldots, P_{n}\right)=\operatorname{MV}\left(P_{1}, \ldots, P_{k}\right) \cdot \operatorname{MV}\left(\bar{P}_{k+1}, \ldots, \bar{P}_{n}\right),
$$

where $\bar{P}_{i}$ is the image of $P_{i}$ under the projection along $L$.

Here is the (inductive) characterization of families with mixed volume one.

Theorem 19 (Esterov, Gusev '12). Let $P_{1}, \ldots, P_{n}$ be lattice polytopes in $\mathbb{R}^{n}$.

Then $\operatorname{MV}\left(P_{1}, \ldots, P_{n}\right)=1$ if and only if $\mathrm{MV}\left(P_{1}, \ldots, P_{n}\right) \neq 0$, and there exists an integer $1 \leq k \leq n$ such that, up to translations, $k$ of the polytopes are faces of the same unimodular $k$-simplex $Q$, and the projection of the other $n-k$ simplices along $Q$ form a family of mixed volume one.

Note that the 'if'-direction follows from Lemmas 17 and 18 together with the monotonicity of the mixed volume, while the 'only if'-direction is a highly non-trivial result.

For the proof of Theorem 5, we need the following simple observation.

Lemma 20. Let $P_{1}, \ldots, P_{k}$ be faces of $\Delta_{n}=\operatorname{conv}\left(0, e_{1}, \ldots, e_{n}\right)$ such that $\operatorname{dim}\left(P_{I}\right) \geq|I|$ for any $\emptyset \neq I \subseteq[k]$. Then $\operatorname{int}_{\mathbb{Z}}\left(P_{I}\right)=\emptyset$ for any $\emptyset \neq I \subseteq[k]$.

Proof. Let $\emptyset \neq I \subseteq[k]$, and $j:=|I|$. We note that $P_{I} \subseteq j \Delta_{n}$. Hence, there exists a unique face $F$ of $j \Delta_{n}$ such that $\operatorname{int}\left(P_{I}\right) \subseteq \operatorname{int}(F)$. Let $d:=\operatorname{dim}(F)$, so $F \cong j \Delta_{d}$. Since by assumption $1 \leq j \leq \operatorname{dim}\left(P_{I}\right) \leq d$, we get $\operatorname{int}_{\mathbb{Z}}\left(j \Delta_{d}\right)=\emptyset$, hence, $\operatorname{int}_{\mathbb{Z}}\left(P_{I}\right)=\emptyset$. 
Proof of Theorem 5. The direction (1) $\Rightarrow$ (2) follows directly from Corollary 14 . For $(2) \Rightarrow(1)$, we can assume by Theorem 19 and Lemma 17 that $P_{1}, \ldots, P_{k}$ (for some $1 \leq k \leq n$ ) are faces of the unimodular simplex $\Delta_{k} \subset \mathbb{R}^{k}$, $\operatorname{dim}\left(P_{[k]}\right)=k$, and $\operatorname{dim}\left(P_{I}\right) \geq|I|$ for any $\emptyset \neq I \subseteq[k]$. In particular, Lemma 20 yields $\operatorname{int}_{\mathbb{Z}}\left(P_{I}\right)=\emptyset$ for any $\emptyset \neq I \subseteq[k]$. This proves the statement for $k=n$, so let $k<n$. Considering the projection along $\mathbb{R}^{k}$, Theorem 19 implies $\operatorname{MV}\left(\bar{P}_{k+1}, \ldots, \bar{P}_{n}\right)=1$, thus, $\operatorname{md}\left(\bar{P}_{k+1}, \ldots, \bar{P}_{n}\right)=0$ by induction. Let us assume that there exists $\emptyset \neq I \subseteq[n]$ such that $\operatorname{int}_{\mathbb{Z}}\left(P_{I}\right) \neq \emptyset$, in particular, $I \nsubseteq[k]$. We observe that $\bar{P}_{I}$ equals $\bar{P}_{I \cap\{k+1, \ldots, n\}}$ up to a translation. Hence, $\operatorname{int}_{\mathbb{Z}}\left(\bar{P}_{I \cap\{k+1, \ldots, n\}}\right) \neq \emptyset$, a contradiction to $\operatorname{md}\left(\bar{P}_{k+1}, \ldots, \bar{P}_{n}\right)=0$.

Remark 21. Let us note the following observation: If $P_{1}, \ldots, P_{k}$ are in $\mathbb{R}^{n}$ such that $\operatorname{mcd}\left(P_{1}, \ldots, P_{k}\right)=k+1$, then we have $k \leq \operatorname{dim}\left(P_{[k]}\right)$ by nonnegativity of the mixed degree.

Proof of Theorem 8. We first consider the case $m=n+1$. Here, we have $\operatorname{int}_{\mathbb{Z}}\left(P_{I}\right)=\emptyset$ for any $\emptyset \neq I \subsetneq[n+1]$. Since as in the proof of Proposition 4 we get $\operatorname{MV}\left(P_{1}, \ldots, P_{n-1}, P_{n}+P_{n+1}\right)=2$, Corollary 14 implies that $\left|\operatorname{int}_{\mathbb{Z}}\left(P_{[n+1]}\right)\right|=1$. By a well-known result in the geometry of numbers [21], there are up to unimodular equivalence only a finite number of lattice polytopes with one interior lattice point in fixed dimension $n$. This implies that there are only finitely many families $P_{1}, \ldots, P_{n+1}$ with $\operatorname{md}\left(P_{1}, \ldots, P_{n+1}\right)=0$ up to our identification.

So, let $m>n+1$. Remark 21 applied to $P_{1}, \ldots, P_{n} \operatorname{implies} \operatorname{dim}\left(P_{[n]}\right)=n$, so $P_{1}, \ldots, P_{n+1}$ is proper. Let us fix $P_{1}, \ldots, P_{n+1}$ as one of the finitely many types in the above argument. Let $n+1<i \leq m$. By similarly considering $P_{1}, \ldots, P_{n}, P_{i}$ we deduce that there are only finitely many possibilities (say, $N$ many) for $P_{i}$ up to translation. Note that $N$ only depends on $n$.

Hence, we may assume that $m>n+1+(n-1) N$. By the pigeonhole principle, there exist $P_{i_{1}}, \ldots, P_{i_{n}}$ (with $n+2 \leq i_{1}<\cdots<i_{n} \leq n+2+$ $(n-1) N)$ that are all equal to the same lattice polytope $Q$ up to translations. Again, Remark 21 applied to $P_{i_{1}}, \ldots, P_{i_{n}}$ yields that $\operatorname{dim}(Q)=n$. Moreover, Corollary 14 implies that $\operatorname{Vol}(Q)=\operatorname{MV}(Q, \ldots, Q)=1$, i.e., $Q$ is a unimodular $n$-simplex.

Let $i \in[m] \backslash\left\{i_{1}, \ldots, i_{n}\right\}$ such that $P_{i}$ is not contained in $Q$ up to translations. We will show that this case cannot occur. Again, Corollary 14 yields $\operatorname{MV}\left(Q, \ldots, Q, P_{i}\right)=1$ (where $Q$ is chosen $n-1$ times). Now, $\operatorname{since} \operatorname{dim}(Q)=n$, Theorem 19 implies that there exists $1 \leq k \leq n$ such that $k$ of the polytopes $Q, \ldots, Q, P_{i}$ are contained up to translations in a $k$-dimensional unimodular simplex $S$ and the projection of the other $(n-k)$ polytopes along this simplex yields again a family of mixed volume one. Assume $k>1$. In this case, one of the $Q$ 's would be contained in $S$ up to translation, hence $S$ would be equal to $Q$ up to translation, so $k=n$, and $P_{i}$ would be contained in $Q$ up to translation, a contradiction. Therefore, $k=1$, and $P_{i}$ is contained in $S$ up to translation. Since $P_{i}$ is not a point, we see that $P_{i}=S$ must be a lattice interval containing two lattice points. Since projecting $Q, \ldots, Q$ along $P_{i}$ (via a lattice projection $\pi_{i}$ ) yields again a family of full-dimensional lattice polytopes of mixed volume 
one, Proposition 6 implies that $\pi_{i}(Q)$ is an $(n-1)$-dimensional unimodular simplex. In particular, we see that there must be two vertices of $Q$ that get mapped to the same vertex of $\pi_{i}(Q)$. Hence, since $P_{i}$ lies in a fiber of $\pi_{i}$, we deduce that $P_{i}$ is up to a translation an edge of $Q$, again a contradiction.

Finally, let us consider the situation that all lattice polytopes are contained in a unimodular $n$-simplex $Q$ up to translations. Because we have $\operatorname{codeg}\left(\Delta_{j}\right)=j+1$, no face of $Q$ of dimension $j<n$ can appear $j+1$ times. This proves the last statement in the theorem. It remains to observe the following easily verified binomial identity

$$
\sum_{i=1}^{n-1} i\left(\begin{array}{c}
n+1 \\
i+1
\end{array}\right)=\left(2^{n}-1\right)(n-1) .
$$

\subsection{Mixed Degree at Most One}

Let $P_{1}, \ldots, P_{m}$ be lattice polytopes in $\mathbb{R}^{n}$. Let us define for $\emptyset \neq I \subseteq[m]$

$$
g(I):=\sum_{\emptyset \neq J \subseteq I}(-1)^{|I|-|J|}\left|\operatorname{int}_{\mathbb{Z}}\left(P_{J}\right)\right| .
$$

Theorem 22 (Khovanskii' 78, Bihan '14). If $P_{1}, \ldots, P_{m}$ are $n$-dimensional lattice polytopes, then $g([\mathrm{~m}])$ is nonnegative.

Bihan's proof $[7$, Theorem 4.15(4)] is purely combinatorial.

Remark 23. Let us assume $m \leq n$, and explain why nonnegativity follows from the algebro-geometric meaning of $g([m])$. Given $P_{1}, \ldots, P_{m}$, these lattice polytopes are the Newton polytopes of generic Laurent polynomials $f_{1}, \ldots, f_{m} \in \mathbb{C}\left[x_{1}^{ \pm}, \ldots, x_{n}^{ \pm}\right]$. We consider the set $X$ of their common solutions in the algebraic torus $\left(\mathbb{C}^{*}\right)^{n}=(\mathbb{C} \backslash\{0\})^{n}$. Let $\bar{X}$ be its Zariski closure in the projective toric variety associated to the normal fan of $P_{[m]}$. Then, $g([m])$ equals the geometric genus of $\bar{X}$ (i.e., $h^{n-m, 0}(\bar{X})$ ), see [20].

Example 24. As we see again from Example 11, the full-dimensionality assumption cannot be removed from Theorem 22. In this situation, we have $\left|\operatorname{int}_{\mathbb{Z}}\left(P_{[2]}\right)\right|=\left|\operatorname{int}_{\mathbb{Z}}\left(P_{1}\right)\right|=0$, while $\left|\operatorname{int}_{\mathbb{Z}}\left(P_{2}\right)\right|$ can be arbitrarily large. Hence, $g([2])$ can be arbitrarily negative.

For $\emptyset \neq I \subseteq[m]$, let us now consider the following variant of $g(I)$ :

$$
\tilde{g}(I):=\sum_{\emptyset \neq J \subsetneq I}(-1)^{|I|-1-|J|}\left|\operatorname{int}_{\mathbb{Z}}\left(P_{J}\right)\right| .
$$

Lemma 25. For $\emptyset \neq I \subseteq[m]$,

$$
\tilde{g}(I)=\sum_{\emptyset \neq J \subsetneq I} g(J) .
$$


Proof. Möbius-inversion states that

$$
\left|\operatorname{int}_{\mathbb{Z}}\left(P_{I}\right)\right|=\sum_{\emptyset \neq J \subseteq I} g(J) .
$$

Therefore, the statement follows from $\tilde{g}(I)=\left|\operatorname{int}_{\mathbb{Z}}\left(P_{I}\right)\right|-g(I)$.

We can now give the combinatorial proof of Soprunov's lower bound theorem and the characterization of its equality case.

Proof of Theorem 10 and Proposition 12. Let $P_{1}, \ldots, P_{n}$ be $n$-dimensional lattice polytopes. In this case, Theorem 22 implies $g(I) \geq 0$ for any $\emptyset \neq I \subseteq[n]$. Hence, Lemma 25 yields $\tilde{g}([n]) \geq 0$. Now, rewriting Corollary 14 yields

$$
\operatorname{MV}\left(P_{1}, \ldots, P_{n}\right)-1=g([n])=\left|\operatorname{int}_{\mathbb{Z}}\left(P_{[n]}\right)\right|-\tilde{g}([n]) \leq\left|\operatorname{int}_{\mathbb{Z}}\left(P_{[n]}\right)\right| .
$$

In particular, we have equality if and only if $\tilde{g}([n])=0$. By Lemma 25 this is equivalent to $g(I)=0$ for all $\emptyset \neq I \subsetneq[n]$. By (2), this just means that $\left|\operatorname{int}_{\mathbb{Z}}\left(P_{I}\right)\right|=0$ for any $\emptyset \neq I \subsetneq[n]$ which is equivalent to mixed degree $\leq 1$.

\section{Acknowledgements}

Open Access funding provided by Projekt DEAL. The author would like to thank Alan Stapledon, Frédéric Bihan, Alexander Esterov, Alicia Dickenstein, Christian Haase, Raman Sanyal, and especially Ivan Soprunov for useful discussions and helpful remarks. The author is grateful to the MSRI and the Fields Institute for financial support. First ideas about a mixed degree go back to the stay of the author as a postdoctoral fellow at the MSRI as part of the program on Tropical Geometry. This paper was essentially finished during a stay at the Fields Institute as part of the program on Combinatorial Algebraic Geometry. The author is a PI in the Research Training Group Mathematical Complexity Reduction funded by the German Research Foundation (DFG-GRK 2297), and is also partially supported by the Vetenskapsrådet grant NT:2014-3991 (as an affiliated researcher with Stockholm University). Finally, the author thanks the anonymous referees for careful corrections and suggestions regarding the presentation of the paper.

Open Access. This article is licensed under a Creative Commons Attribution 4.0 International License, which permits use, sharing, adaptation, distribution and reproduction in any medium or format, as long as you give appropriate credit to the original author(s) and the source, provide a link to the Creative Commons licence, and indicate if changes were made. The images or other third party material in this article are included in the article's Creative Commons licence, unless indicated otherwise in a credit line to the material. If material is not included in the article's Creative Commons licence and your intended use is not permitted by statutory regulation or exceeds the permitted use, you will need to obtain permission directly from the copyright holder. To view a copy of this licence, visit http://creativecommons.org/ licenses/by/4.0/.

Publisher's Note Springer Nature remains neutral with regard to jurisdictional claims in published maps and institutional affiliations. 


\section{References}

[1] Araujo, C., and Monsôres, D. On smooth lattice polytopes with small degree. Comm. Algebra 44, 2 (2016), 500-514.

[2] Batyrev, V. Dual polyhedra and mirror symmetry for Calabi-Yau hypersurfaces in toric varieties. J. Algebraic Geom. 3, 3 (1994), 493-535.

[3] Batyrev, V., and Borisov, L. On Calabi-Yau complete intersections in toric varieties. In Higher-dimensional complex varieties (Trento, 1994) (1996), de Gruyter, Berlin, pp. 39-65.

[4] Batyrev, V., and Nill, B. Multiples of lattice polytopes without interior lattice points. Mosc. Math. J. 7, 2 (2007), 195-207, 349.

[5] Batyrev, V., and Nill, B. Combinatorial aspects of mirror symmetry. In Integer points in polyhedra-geometry, number theory, representation theory, algebra, optimization, statistics, vol. 452 of Contemp. Math. Amer. Math. Soc., Providence, RI, 2008, pp. 35-66.

[6] Beck, M., Nill, B., Reznick, B., Savage, C., Soprunov, I., and Xu, Z. Let me tell you my favorite lattice-point problem .... In Integer points in polyhedrageometry, number theory, representation theory, algebra, optimization, statistics, vol. 452 of Contemp. Math. Amer. Math. Soc., Providence, RI, 2008, pp. 179187.

[7] Bihan, F. Irrational Mixed Decomposition and Sharp Fewnomial Bounds for Tropical Polynomial Systems. Discrete Comput. Geom. 55, 4 (2016), 907-933.

[8] Blanco, M., and Santos, F. Lattice 3-polytopes with few lattice points. SIAM J. Discrete Math. 30, 2 (2016), 669-686.

[9] Cattani, E., Cueto, M. A., Dickenstein, A., Di Rocco, S., and Sturmfels, B. Mixed discriminants. Math. Z. 274, 3-4 (2013), 761-778.

[10] Cox, D., and Dickenstein, A. Codimension theorems for complete toric varieties. Proc. Am. Math. Soc. 133, 11 (2005), 3153-3162.

[11] Danilov, V. I., and Khovanskiı̌, A. G. Newton polyhedra and an algorithm for calculating Hodge-Deligne numbers. Izv. Akad. Nauk SSSR Ser. Mat. 50, 5 (1986), 925-945.

[12] Di Rocco, S., Haase, C., Nill, B., and Paffenholz, A. Polyhedral adjunction theory. Algebra Number Theory 7, 10 (2013), 2417-2446.

[13] Dickenstein, A., Di Rocco, S., and Piene, R. Classifying smooth lattice polytopes via toric fibrations. Adv. Math. 222, 1 (2009), 240-254.

[14] Dickenstein, A., and Nill, B. A simple combinatorial criterion for projective toric manifolds with dual defect. Math. Res. Lett. 17, 3 (2010), 435-448.

[15] Esterov, A., and Gusev, G. Systems of equations with a single solution. J. Symbolic Comput. 68 (2015), 116-130. 
[16] Haase, C., Juhnke-Kubitzke, M., Sanyal, R., and Theobald, T. Mixed Ehrhart polynomials. Electron. J. Combin. 24, 1 (2017), Paper 1.10, 14.

[17] Haase, C., Nill, B., and Payne, S. Cayley decompositions of lattice polytopes and upper bounds for $h^{*}$-polynomials. J. Reine Angew. Math. 637 (2009), 207-216.

[18] Jochemko, K., and Sanyal, R. Combinatorial mixed valuations. Adv. Math. 319 (2017), 630-652.

[19] Katz, E., and Payne, S. Piecewise polynomials, Minkowski weights, and localization on toric varieties. Algebra Number Theory 2, 2 (2008), 135-155.

[20] Khovanskiü, A. G. Newton polyhedra, and the genus of complete intersections. Funktsional. Anal. i Prilozhen. 12, 1 (1978), 51-61.

[21] Lagarias, J. C., and Ziegler, G. M. Bounds for lattice polytopes containing a fixed number of interior points in a sublattice. Canad. J. Math. 43, 5 (1991), 1022-1035.

[22] Nill, B. Lattice polytopes having $h^{*}$-polynomials with given degree and linear coefficient. European J. Combin. 29, 7 (2008), 1596-1602.

[23] Nill, B., and Padrol, A. The degree of point configurations: Ehrhart theory, Tverberg points and almost neighborly polytopes. European J. Combin. 50 (2015), 159-179.

[24] Nill, B., and Ziegler, G. M. Projecting lattice polytopes without interior lattice points. Math. Oper. Res. 36, 3 (2011), 462-467.

[25] Schneider, R. Convex bodies: the Brunn-Minkowski theory, vol. 44 of Encyclopedia of Mathematics and its Applications. Cambridge University Press, Cambridge, 1993.

[26] Soprunov, I. Global residues for sparse polynomial systems. J. Pure Appl. Algebra 209, 2 (2007), 383-392.

[27] Steffens, R., and Theobald, T. Combinatorics and genus of tropical intersections and Ehrhart theory. SIAM J. Discrete Math. 24, 1 (2010), 17-32.

Benjamin Nill

Fakultät für Mathematik

Otto-von-Guericke-Universität Magdeburg

Universitätsplatz 2

39106 Magdeburg

Germany

e-mail: benjamin.nill@ovgu.de

Received: 10 February 2018.

Accepted: 8 June 2019. 